Portland State University

PDXScholar

Winter 3-20-2015

\title{
Performance Metrics for Depth-based Signal Separation Using Deep Vertical Line Arrays
}

John K. Boyle

Portland State University

Follow this and additional works at: https://pdxscholar.library.pdx.edu/open_access_etds

Part of the Oceanography Commons, and the Signal Processing Commons Let us know how access to this document benefits you.

\section{Recommended Citation}

Boyle, John K., "Performance Metrics for Depth-based Signal Separation Using Deep Vertical Line Arrays" (2015). Dissertations and Theses. Paper 2198.

https://doi.org/10.15760/etd.2195

This Thesis is brought to you for free and open access. It has been accepted for inclusion in Dissertations and Theses by an authorized administrator of PDXScholar. Please contact us if we can make this document more accessible: pdxscholar@pdx.edu. 


\title{
Performance Metrics for Depth-based Signal Separation Using Deep Vertical Line Arrays
}

John K. Boyle

A thesis submitted in partial fulfillment of the requirements for the degree of

\author{
Master of Science \\ in \\ Electrical and Computer Engineering
}

Thesis Committee:

T. Martin Siderius, Chair

Lisa M. Zurk

Richard L. Campbell

Portland State University

2015 
(C) 2015 John K. Boyle 


\begin{abstract}
Vertical line arrays (VLAs) deployed below the critical depth in the deep ocean can exploit reliable acoustic path (RAP) propagation, which provides low transmission loss (TL) for targets at moderate ranges, and increased TL for distant interferers. However, sound from nearby surface interferers also undergoes RAP propagation, and without horizontal aperture, a VLA cannot separate these interferers from submerged targets. A recent publication by McCargar and Zurk (2013) addressed this issue, presenting a transform-based method for passive, depth-based separation of signals received on deep VLAs based on the depth-dependent modulation caused by the interference between the direct and surface-reflected acoustic arrivals. This thesis expands on that work by quantifying the transform-based depth estimation method performance in terms of the resolution and ambiguity in the depth estimate. Then, the depth discrimination performance is quantified in terms of the number of VLA elements.
\end{abstract}




\section{Acknowledgements}

The computer codes used for the full ocean acoustic environment simulations in this thesis are extensions of a code originally developed by Reid McCargar in 2012. Also, the wind noise simulations were possible because of a MATLAB wrapper for OASN provided by John Gebbie.

I would like to thank my advisor, Martin Siderius, for encouraging me to study at Portland State University, and for supporting my interests in classes and in research. He has taught me how to simplify and accelerate my research efforts.

I would like to thank Lisa Zurk for inviting me to work on this project. I have learned dedication, scholarship, and excellence in research from her example.

I also thank Gabe Kniffin and Scott Schecklman for their input, ideas, and partnership in this project; I've had a lot of fun working with them.

This work was supported by the office of Naval Research. 


\section{Table of Contents}

Abstract $\quad$ i

Acknowledgements $\quad$ ii

List of Tables $\quad$ iv

List of Figures $\quad$ v

1 Introduction 1

1.1 Executive Summary . . . . . . . . . . . . . . . . . 1

1.2 Thesis Contributions . . . . . . . . . . . . . . . . . . 2

2 Background $\quad 4$

2.1 Introduction . . . . . . . . . . . . . . . . . 4

2.2 Conventional Plane-wave beamformer . . . . . . . . . . . . . . . 5

2.3 Signals received by deep receivers . . . . . . . . . . . . . . . . . 9

2.3.1 Basic Acoustic Propagation in Deep Ocean . . . . . . . . . . . 9

2.3.2 Noise and Interferers . . . . . . . . . . . . . . . . . . . . 13

2.3.3 Simulation of VTR from Deep VLA . . . . . . . . . . . . . . . 14

2.4 Literature Review of Source Localization Methods . . . . . . . . . . . 17

2.5 Conclusion . . . . . . . . . . . . . . . . . 19

3 Depth-Harmonic Structure Theory $\quad 21$

4 Transform-Based Depth Estimation Performance 29

4.1 Track Extent and Depth Resolution . . . . . . . . . . . . . . . . 31

4.2 Trace Signal Sampling and Depth Ambiguity . . . . . . . . . . . . . 33

5 Estimating Depth by Measuring Null Spacing 37

5.1 Aperture Requirements . . . . . . . . . . . . . . . . 38

6 Summary 41

$\begin{array}{ll}\text { Bibliography } & 43\end{array}$ 


\section{List of Tables}

2.1 Simulation parameters used to create Fig. 2.8. . . . . . . . . . 15

3.1 Normal mode (KRAKEN) simulation parameters used in generating VTR data shown in Figure 3.2. . . . . . . . . . . . . . . . 25

4.1 Alias (effective Nyquist) depths for the target traces corresponding to the trace signals in Figure 3.3. . . . . . . . . . . . . . . . . 


\section{List of Figures}

2.1 Simulated, normalized beamformer output for a uniform line array of 10 elements at half-wavelength spacing. A source is located at $30^{\circ}$, and a corresponding peak is seen in the output. . . . . . . . . . . . .

2.2 Simulated, normalized VTR for a 10 element array at half-wavelength element spacing, and a source that moves at constant radial distance to the array through angles of $+45^{\circ}$ to $-45^{\circ} \ldots . . . . . .$. . .

2.3 Normal mode simulation of acoustic transmission loss for a receiver at the depth of the sound speed minimum in the deep ocean $(1300 \mathrm{~m})$. . 10

2.4 Normal mode simulation of acoustic transmission loss for a receiver at 100m depth. . . . . . . . . . . . . . . . 11

2.5 Simulated VTR using normal modes for a normalized point source at 100 meters moving past an array at 200 meters. . . . . . . . . . . . . . 12

2.6 The general geometry for received passive signals on a deep vertical line array . . . . . . . . . . . . . . . . . .

2.7 Normal mode simulation of acoustic transmission loss for a deep receiver at 4950 meters. . . . . . . . . . . . . . . . . . . . .

2.8 Simulated beam output at $200 \mathrm{~Hz}$ with a submerged target, a nearby surface interferer, distant shipping noise, wind noise and white noise .

3.1 The general geometry for received passive signals on a deep vertical line array . . . . . . . . . . . . . . . . . . . . . .

3.2 Example of depth-dependent modulation in a VTR at $150 \mathrm{~Hz}$ for source depth of $1 \mathrm{~m}$ (a) and $50 \mathrm{~m}$ (b). The null spacing is proportional to frequency and depth (Eq. 3.2). . . . . . . . . . . . . . . . 24

3.3 Example of depth-dependent modulation in simulated trace signals . $\quad 25$

3.4 Example of transform-based depth estimation . . . . . . . . . . 27

4.1 Simulated VTR showing relationship between extent in time and extent in $\sin \theta \ldots \ldots \ldots$. . . . . . . . . . . . . . . . . . . . . . . . . 33

4.2 Depth transform outputs for three trace signal extents . . . . . . . . 34

4.3 Diagram illustrating effect of irregular sampling $\sin \theta$ on depth transform output. . . . . . . . . . . . . . . . . . . 
5.1 Worst-case analysis of array aperture requirement using 3-dB beamwidth as the null location error . . . . . . . . . . . . . . .

5.2 Error in depth estimate using a null location error equal to the 3 - $\mathrm{dB}$ beamwidth . . . . . . . . . . . . . . . 


\section{Chapter 1}

\section{Introduction}

\subsection{Executive Summary}

At low frequencies, the primary impediment to the detection of quiet submerged sources is the ambient noise due to surface ship traffic (Wenz, 1962). However, recent work has shown that vertical line arrays (VLAs) deployed below the critical depth the depth below the channel axis at which the sound speed is equal to the maximum sound speed near the surface - may benefit from propagation effects that significantly reduce distant shipping noise (Gaul et al., 2007, Li et al., 2010). In addition, sound from sources at moderate ranges $(20-35 \mathrm{~km})$ undergo propagation via the reliable acoustic path (RAP) (Urick, 1996), which results in a 10-20 dB reduction in transmission loss (TL) compared to a shallow receiver at the same range (McCargar and Zurk, 2012). However, these favorable propagation conditions also apply to nearby surface interferers, and while a deep VLA with sufficient resolution can separate such moderate-range sources from distant shipping noise in vertical angle, the lack of horizontal aperture prohibits azimuthal rejection. Matched-field methods and adaptive array processing techniques that have been proposed for depth discrimination with 
VLAs to address this challenge are hindered by environmental uncertainty (Baggeroer et al., 1993, Zurk et al., 2003).

One promising alternative takes advantage of the Lloyd's mirror interference pattern that arises from the coherent summation of the direct and surface-reflected acoustic paths. This interference structure introduces depth-harmonic modulation in the signals from submerged targets, providing a means of depth-based signal separation using a modified Fourier transform (McCargar and Zurk, 2012, 2013, Zurk et al., 2013). A more recent method based on an extended Kalman filter also utilizes the observation of the depth-harmonic modulation, but on a single hydrophone (Duan et al., 2014). However, that method uses multiple observations coupled with a motion hypothesis to compensate for the lack of array aperture, and processing gain obtained from a known pseudorandom source signal, which would not apply to passive detection.

\subsection{Thesis Contributions}

In this thesis, results are presented that characterize and quantify the performace of the transform-based depth estimation method developed in previous work (McCargar and Zurk, 2012, 2013, Zurk et al., 2013), but in terms of the characteristics of the depth-harmonic modulation and its sampling via deep VLA. Chapter 2 reviews pertinent theory and literature that is the background for this work, and Ch. 3 reviews the problem geometry, the depth-harmonic modulation, and the transform based depth separation method. Then, the performance of the transform based method is discussed in Ch. 4 in terms of the effect of target track extent and uneven sampling on the resolution and ambiguity (aliasing) in the transform depth estimate. Finally, in Ch. 5 is discussed a simplified method of depth estimation based on direct measure- 
ment of interference structure null spacing. From this, expressions are presented that describe constraints for array apertures that will provide sufficient observation and accurate spatial tracking of the depth-harmonic signal modulation in the beamformer output. 


\section{Chapter 2}

\section{Background}

\subsection{Introduction}

This chapter presents theory and also reviews literature that forms the background material for depth based signal separation and the analysis of it in future chapters. First, the theory of the conventional plane-wave beamformer is presented. Then, the signals that are received on deeply deployed receivers in the deep ocean are described in terms of the submerged targets as well as nearby and distant interferers and also the ambient background noise from natural processes. The propagation physics for these signals is discussed as well as how these signals appear in the beamformer outputs. The final section explores how the received and processed signals may be used to identify separate sources of sound and also estimate the depth of each. Several current techniques for source localization, specifically for depth, will be reviewed and their applicability to the problem at hand will be explored. 


\subsection{Conventional Plane-wave beamformer}

Recordings made with a single omnidirectional hydrophone provide temporal and frequency information about the received signals, but do not immediately provide spatial information about the signal, such as its direction of arrival. However, recordings made on multiple receivers in a known spatial arrangement do provide such information due to time of arrival differences of the signals on each hydrophone. The mathematical signal processing operation of plane-wave beamforming permits the received signals to be spatially separated (filtered).

The simplest spatial arrangement is the uniform line array (ULA), a set of receivers, or array elements, equally spaced in a line. If a signal arrives in a direction parallel to the array (endfire direction), it will be seen on each receiver, but delayed relative to the first by an amount $(n-1) d / c$, where $n$ is the index of the receiver, $d$ the receiver spacing, and $c$ the sound speed. The signals may be combined to spatially favor the endfire direction by delaying each signal by the negative of the travel time delay and then summing. The endfire direction signal is aligned and increases in amplitude, this is array gain. Signals from other directions would have different delays across the array, would not be aligned, and would somewhat cancel each other out in the summation. The process just described is termed delay-and-sum beamforming (Stoica and Moses, 2005).

In the frequency domain, a signal may be expressed as the sum of sinusoids with varying amplitudes and phases. The time delays just discussed become phase delays, where phase refers to the current position of a wave in its oscillation relative to some fixed point along the waveform (for example, a peak or trough or one of the zero- 
points). A signal that is composed of a single tone may be expressed as follows in the time and frequency domains respectively(Stoica and Moses, 2005):

$$
x(t)=A \cos \left(\omega_{\circ} t-\phi\right)=A \cos (\omega(t-n d / c)), \quad X\left(\omega_{\circ}\right)=A e^{i \phi}
$$

where $A$ is the amplitude, $\omega_{o}$ the frequency, the phase offset ("the signal's phase") is $\phi=\omega n d / c=k n d$ where $k$ is the wavenumber, and $X$ denotes the frequency domain representation of the time-domain signal.

Now, assuming a discrete Fourier transform (DFT) has been applied to the received signal, the signal alignment by time delays previously discussed may be applied here with a phase delay, $\phi$. The delay is relative to the top phone of the array (for a VLA) and is expressed in terms of the angle of arrival $\theta$ relative to the perpendicular to the array,

$$
\text { phase delay }=k(n-1) d \sin \theta \text {. }
$$

When $\theta=90^{\circ}$ the delays are maximum across the array, which corresponds to an arrival from the vertical endfire direction. When the wave arrives with angle of zero, the delay is zero; the wave arrives at every element at the same time.

In terms of matrix multiplication operations, a conventional beamformer may be expressed as

$$
B\left(\theta, \omega_{\circ}\right)=\left[\begin{array}{llll}
x_{1, \omega_{\circ}} & x_{2, \omega_{\circ}} & \ldots & x_{n, \omega_{\circ}}
\end{array}\right]\left[\begin{array}{c}
1 \\
e^{-k d \sin \theta} \\
\vdots \\
e^{-k(n-1) d \sin \theta}
\end{array}\right]
$$


where $x_{n, \omega_{o}}$ is the signal on phone $n$ at frequency $\omega_{o}$.

A uniform line array permits spatial separation between sources at different angles relative to the axis of the array. However, a ULA cannot distinguish the direction of a source around the axis of the array. In the case of a vertical ULA, the array lacks azimuthal aperture, but does have aperture in vertical angle.

Also, there is a limit on the minimum separation between sources that may be separated on the beamformer output. The Rayleigh resolution limit (Stoica and Moses, 2005) expresses the minimum angular source separation resolvable by a given ULA of length $L$ with number of elements $N$, spaced at half-wavelength for a frequency $\omega$

$$
\theta_{\min }=\frac{\lambda}{N d}
$$

where $N$ is the number of array elements.

A simulated, normalized, example beamformer output over all angles for a ULA is given in 2.1. A source was placed at $30^{\circ}$ relative to the array and a peak is seen in the beamformer output at $30^{\circ}$. The peak width is proportional to the number of array elements at half-wavelength spacing for the frequency of the source signal.

A moving source may be tracked by the following method. First, the received signal is separated into sections of perhaps several seconds, these are called the data snapshots. The beamformer is applied to each snapshot and the result at each snapshot time is plotted on a 2D plot with axis of time, angle, and the plot color gives beamformer output amplitude, most commonly in decibels, a plot commonly known as a bearingtime record (BTR) (bearing angle is used for ULA placed in the horizontal plane, however in this thesis VTR will be used). Then, as a source moves through different 


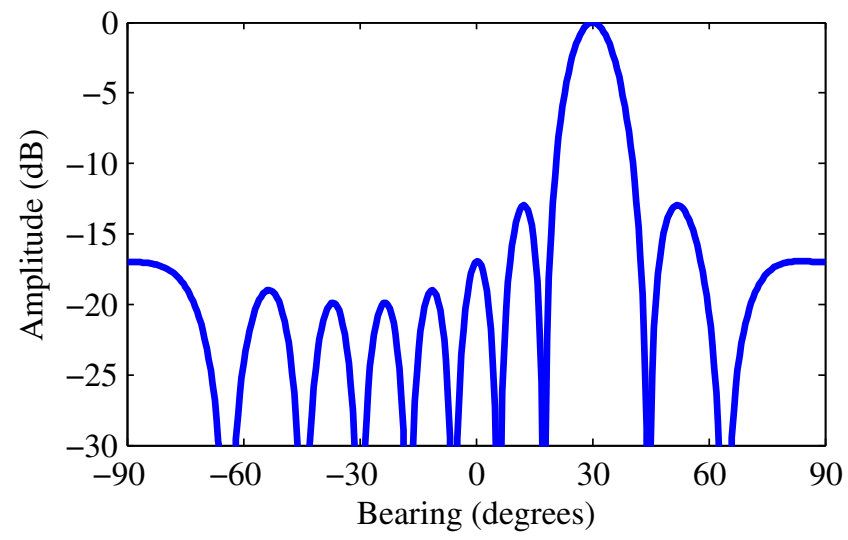

Figure 2.1: Simulated, normalized beamformer output for a uniform line array of 10 elements at half-wavelength spacing. A source is located at $30^{\circ}$, and a corresponding peak is seen in the output.

angles, it will cause a line to be traced in the VTR. An example VTR is shown in Fig. 2.2, which shows the simulated beamformer output as a simulated source moves near a vertical array at a constant radial distance (varying depth and range over time), through the angles $+45^{\circ}$ to $-45^{\circ}$. At evenly spaced time intervals, the signals across the array are computed for the source's current position. The beamformer outputs at each time are plotted side-by-side in the figure with a colormap of thirty dB dynamic range relative to the peak.

A spatially selective time series (here called a trace signal) may be generated by selecting a trace on the VTR that follows the source peak, and then using the trace values (vertical angle as a function of time) to steer the beamformer. The spectrogram of the trace signal is called a scissorgram.

The VTR aids in the detection (due to increased SNR), the tracking, and spatial filtering of spatially distinct signals received on a VLA (and other arrays). However, if two signal traces cross, the signals will be mixed and will perhaps be indistinguishable from one another. This type of interference is nearly unavoidable and is a limitation 


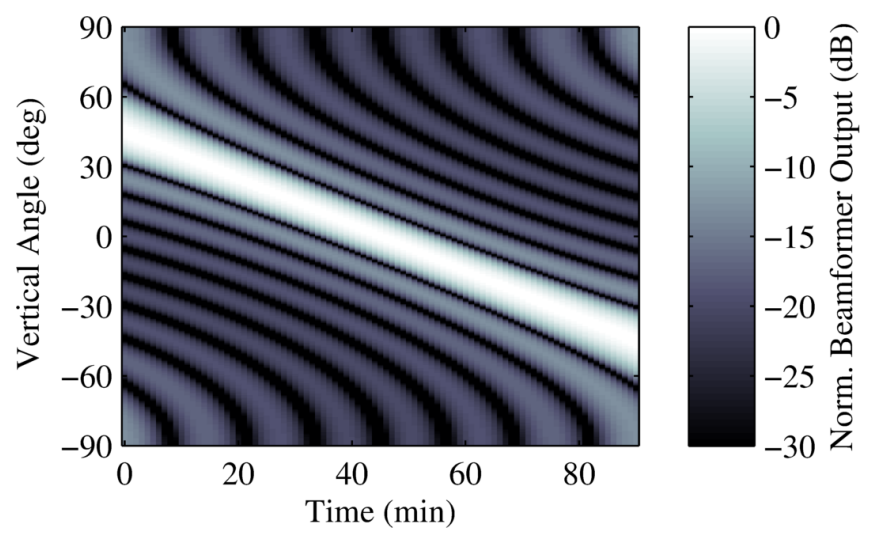

Figure 2.2: Simulated, normalized VTR for a 10 element array at half-wavelength element spacing, and a source that moves at constant radial distance to the array (changing depth and range) through angles of $+45^{\circ}$ to $-45^{\circ}$. The bright line shows the source's path as it moves over time. A path may be selected that follows the source (a VTR trace), and it may be used to produce either an array output time series and spectrogram (scissorgram).

of beamforming.

\subsection{Signals received by deep receivers}

In this section, the acoustic propagation physics of the deep ocean will be discussed, followed by a discussion of the noise and interference received on deep vertical line arrays, and finally the implications of these will be discussed as it relates to identifying surface vs. submerged sources.

\subsubsection{Basic Acoustic Propagation in Deep Ocean}

At low frequencies (up to a few hundred Hertz) surface ships and submarines in the deep ocean may be approximated as point sources. The ocean surface is treated as a perfectly reflecting boundary. The sound speed varies with depth and a typical 


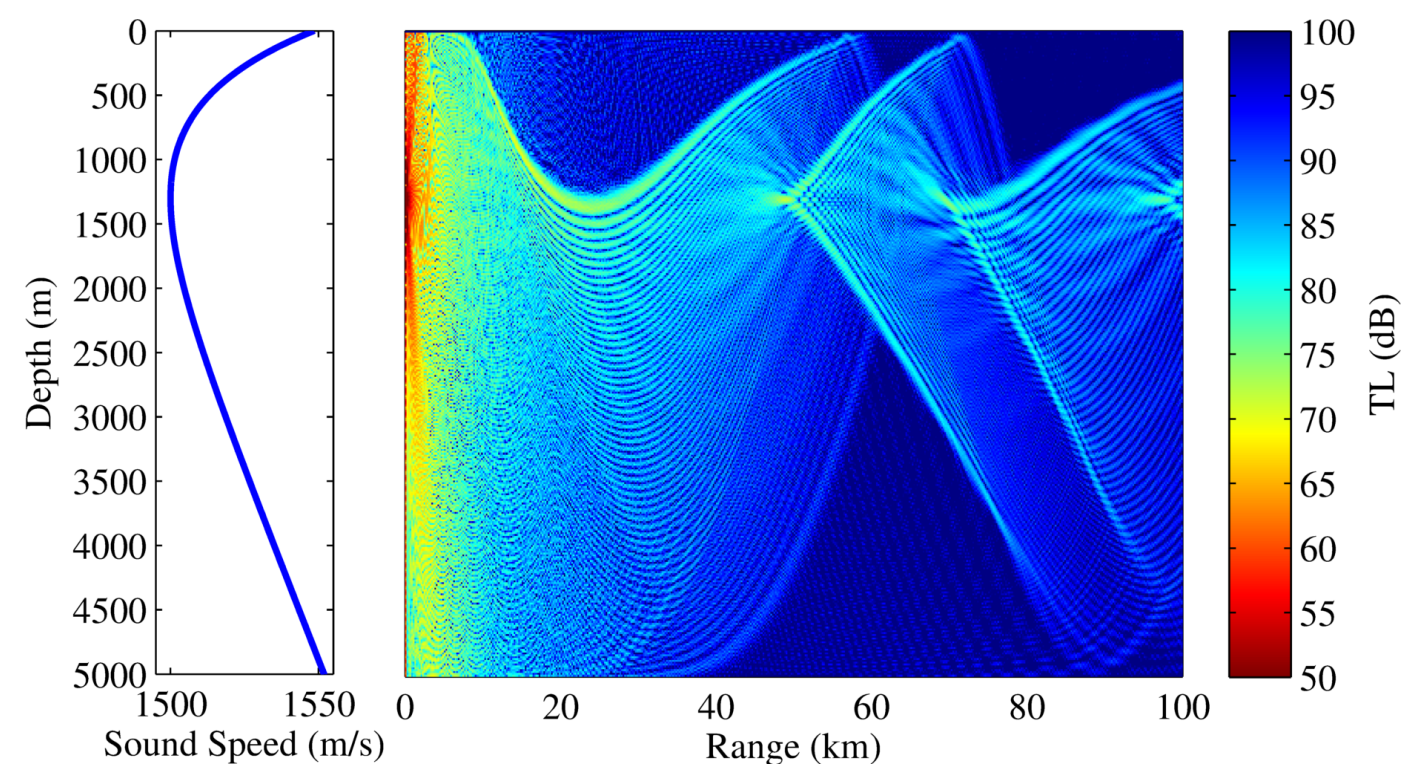

Figure 2.3: Normal mode simulation of acoustic transmission loss for a source or receiver at the depth of the sound speed minimum in the deep ocean $(1300 \mathrm{~m})$. The left plot is the theoretical Munk sound speed profile that shows the key features of sound speed profiles in the deep ocean.

representation of the average sound speed profile of the deep ocean is given by the Munk profile (Munk, 1974), shown in the left hand side of Figure 2.3. The sound speed minimum at about 1300 meters creates what is known as the sound channel, a refracting acoustic duct, seen in Figure 2.3 in the complex pattern to on the right hand side.

The general deep ocean sound speed profile leads to a propagation effect know as convergence zones (Jensen et al., 1997), where sound reaches the surface at certain distances, as seen in the focused areas of lower transmission loss in Figure 2.4 for a source at 100 meters.

Convergence zones typically have a spacing of about 50-70 km. Other sound speed profiles lead to different types of "shadow zones" that may direct sound away from receivers (Brekhovskikh and Lysanov, 2003) and thus complicate detection of quiet 


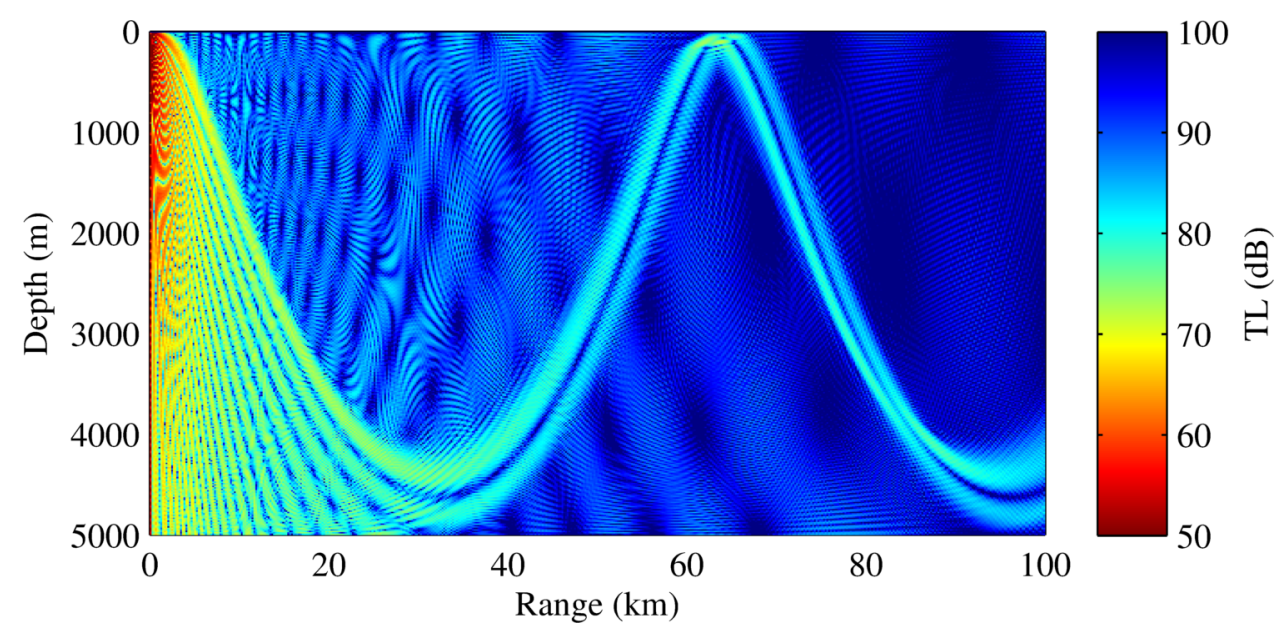

Figure 2.4: Normal mode simulation of acoustic transmission loss for a receiver at 100m depth, with a Munk sound speed profile.

submerged targets. Figure 2.5 show a VTR simulated using normal modes (KRAKEN (Jensen et al., 1997)) with the parameters listed in Table 3.1, except that the source is at 100 meters and the vertical array is centered at 200 meters. There is a strong signal at about $60 \mathrm{~km}$, corresponding to the convergence zone seen in Figure 2.4.

Before $20 \mathrm{~km}$, there are multiple arrivals from the source across a spread of angles. In a shallow water environment, the complex interference between the multipath arrivals is used in waveguide invariant based ranging (D'Spain and Kuperman, 1999), where interference lines in a spectrogram of signals from a distant source may be related to the source's radial motion and range.

A different source-receiver configuration that avoids some of these complications is the general geometry shown in Figure 2.6. The VLA is placed at a depth greater than that of the critical depth of the sound speed profile, defined as the depth at which the sound speed surpasses the highest value at depths above the sound speed minimum. This geometry supports acoustic propagation known as the Reliable Acoustic Path (Urick, 1996), that for nearby sources $(<15 \mathrm{~km})$ provides mid-to-high angle arrivals 


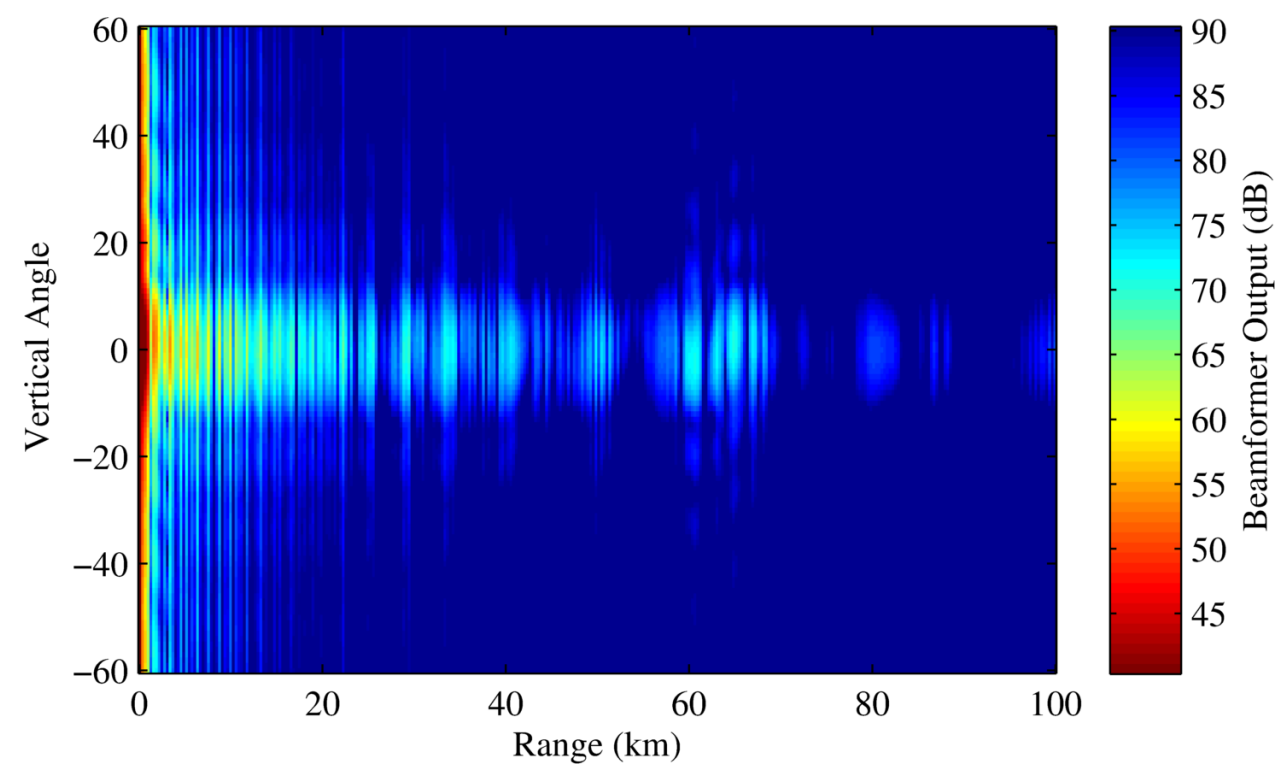

Figure 2.5: Simulated VTR using normal modes for a source at 100 meters moving past an array at centered at 200 meters. All other parameters are as listed in Table 3.1. This can be considered as a spatial sampling of the transmission loss at 100 meters in Figure 2.4. The convergence zone propagation causes the strong signal at about $60 \mathrm{~km}$.

that travel down through the sound speed profile, refracting only a small amount. Sound from distant surface sources arrives near broadside. Transmission loss for this geometry is shown in Figure 2.7.

In Fig. 2.7, it can be seen that for a shallow source at ranges up to about $20 \mathrm{~km}$, transmission loss is 10-15 dB less than for a source in the source-receiver geometry of Fig. 2.4. This region of lower TL in the RAP geometry is favorable for detecting quiet sources but equally favors loud surface interferers (generally shipping vessels).

The sound from submerged sources at the deep VLA is primarily a combination of the direct and surface-reflected paths. These are not readily separable for use in passive, multi-path ranging because the angular separation between them for this geometry is a degree or less, which below the resolution limit (previously discussed) for arrays 


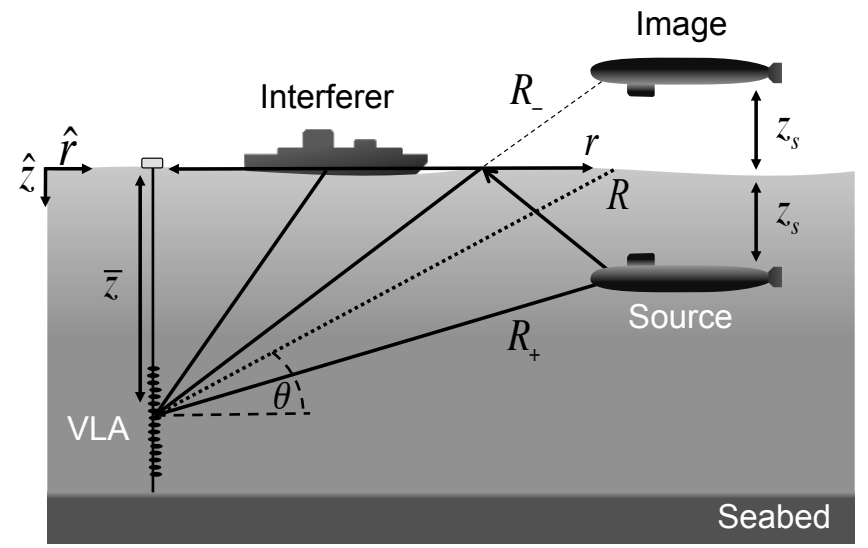

Figure 2.6: The general geometry for received passive signals on a deep vertical line array (not shown to scale). Sound from a submerged source at surface range $r$ and depth $z_{s}$ arrives at an angle of $\theta$ relative to broadside of the VLA whose center lies at depth $\bar{z}$. From image theory, the direct and surface-reflected arrivals correspond to slant ranges $R_{+}$and $R_{-}$, respectively, with slant range $R$ corresponding to the midpoint between the source and its image. Schematic based on Fig. 1 of McCargar and Zurk (2013)

with a reasonable number of elements. As the acoustic path length difference becomes multiples of $\pi$ radians, destructive interference occurs and the received signal exhibits an interference pattern that is proportional to the target's depth. This may be used to estimate depth of sources and thereby distinguish between surface and submerged sources. This will be discussed in detail in the next chapter.

\subsubsection{Noise and Interferers}

On a receiver below the critical depth in the sound speed profile, the low frequency surface ship ambient noise level may be decreased by 10-15 dB, as much of the noise becomes trapped in the ocean duct above the critical depth (Gaul et al., 2007). Much of the noise that does arrive on the array is at broadside and is from distant sources (Li et al., 2010), and noise at high angles is surface noise from waves and wind. That 


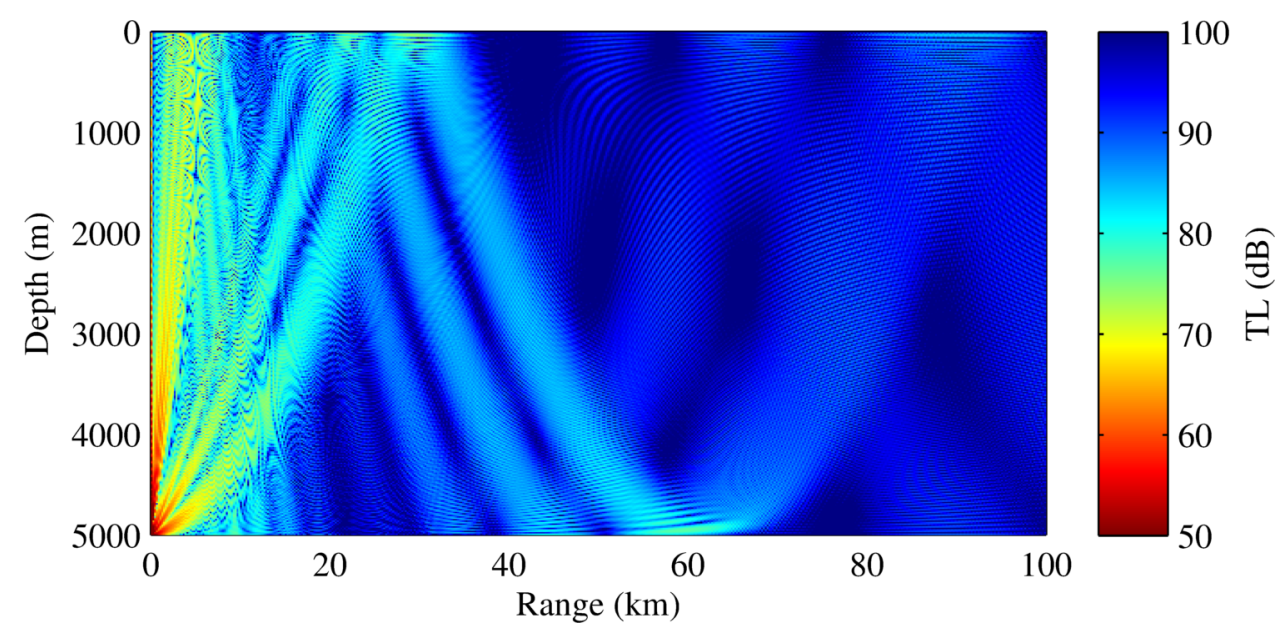

Figure 2.7: Normal mode simulation of acoustic transmission loss for a deep receiver at 4950 meters.

distant noise arrives at broadside is due to the fact that such noise is travelling via refracting paths in the ocean and can only arrive at that depth if it is at or near a turning point in its ray path. Thus the sound speed profile provides a spatial filter, placing much of the background noise away from the higher angles where nearby sources are seen. (Note: this is similar in concept to the spatial filtering in the noise notch (Belousov and Furduev, 1988) in the sound channel, though the directions of quiet and noise are opposite).

\subsubsection{Simulation of VTR from Deep VLA}

A simulated VTR that includes wind noise, isotropic white noise, nearby and distant interferers, and a submerged target is shown in Figure 2.8 (Zurk et al., 2013). In addition to isotropic white noise, wind noise was simulated by random realizations drawn from a wind noise covariance matrix computed by OASES-OASN (Schmidt, 2011). Distant shipping noise was simulated with a random distribution of point sources (Li et al., 2010), and all parameters values are given in Table 2.1. The target 
Table 2.1: Simulation parameters used to create Fig. 2.8.

\begin{tabular}{l|l|l|l}
\hline & $\begin{array}{l}\text { Nearby } \\
\text { Interferer }\end{array}$ & $\begin{array}{l}\text { Distant In- } \\
\text { terferers }\end{array}$ & Target \\
\hline$N_{\text {sources }}$ & 2 & 50 & 1 \\
\hline CPA range (km) & 10,2 & $100-1600$ & 2 \\
\hline CPA time (min) & $-20,80$ & - & 35 \\
\hline Speed (m/s) & 10,13 & - & 5 \\
\hline Depth $(\mathrm{m})$ & 12,15 & $1-10$ & 50 \\
\hline $\begin{array}{l}200 \quad \mathrm{~Hz} \text { source } \\
\text { level (dB re } \\
1 \mu P a)\end{array}$ & 155,160 & 160 & 110 \\
\hline \multicolumn{4}{|c}{ Noise Levels } \\
\hline Isotropic White Noise & $40 \mathrm{~dB}$ \\
\hline
\end{tabular}

and interferer were both assumed to move in a straight line at constant speed between snapshots, with the kinematics listed.

The beam output is shown in Fig. 2.8. The surface ship and the submerged target tracks are visible in the beam output in (a), and were overlaid with colored lines and labeled in (b). The submerged target direct arrival (under the red line in (b)) exhibits a modulation due to the interference of the direct and surface-reflected arrivals, and the surface interferer track also exhibits modulation, though with wider spacing between nulls (as expected for shallower source). Note that a modulation is also present in the surface interferer multipath tracks, but with different null spacing than in the direct arrival, an effect caused by multiple bottom/surface interactions.

A deeply deployed array is well suited for detection of quiet, nearby sources due to spatial separated distant noise, as well as the reduced transmission loss for nearby sources, and the minimally refracted, direct acoustic arrivals. In the next section, several current methods of source localization are reviewed and their potetional utility 

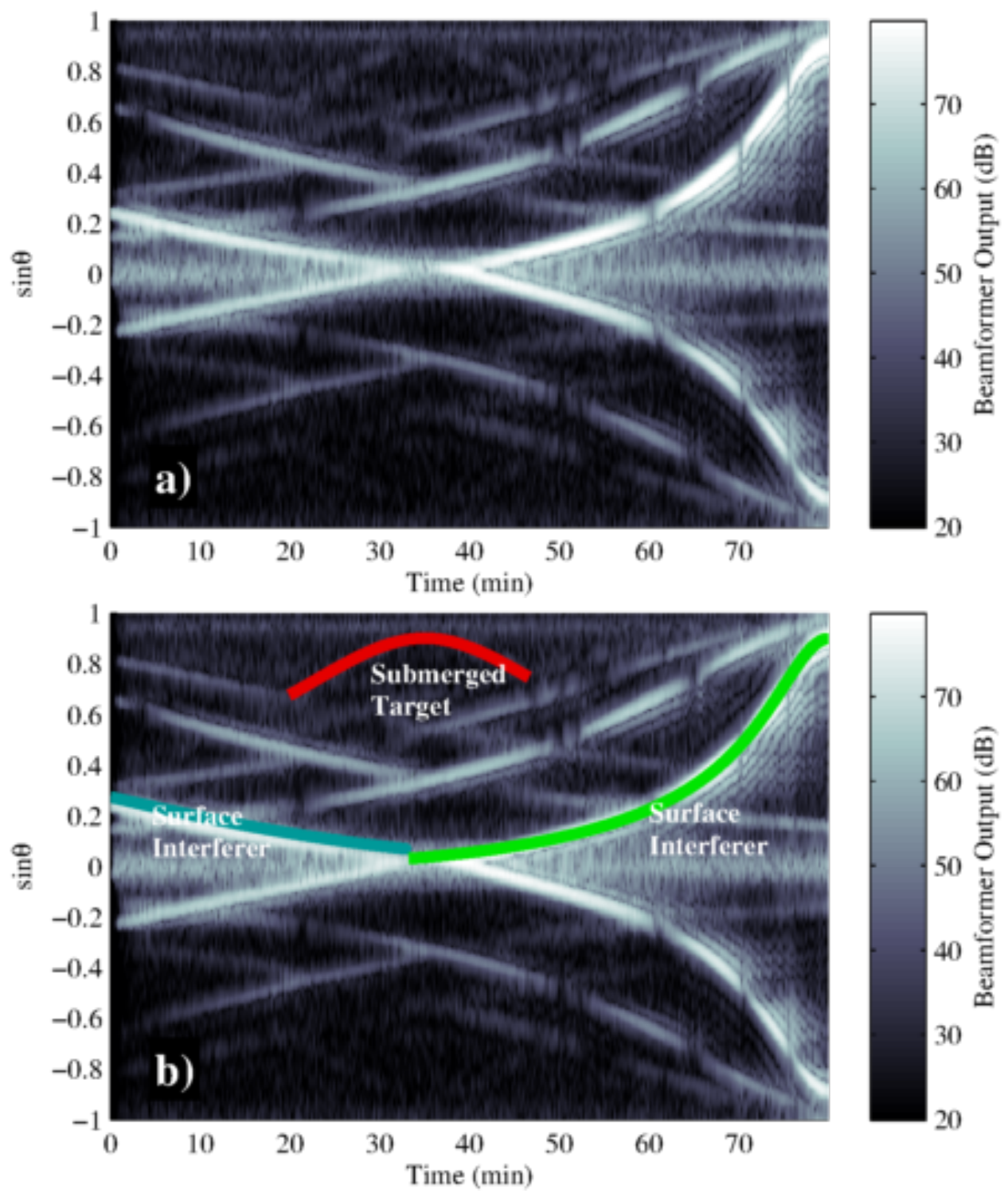

Figure 2.8: Simulated beam output at $200 \mathrm{~Hz}$ with a submerged target, two nearby surface interferers, distant shipping noise, wind noise, and white noise (the only difference between (a) and (b) is the trace overlays). In (b) the blue and green overlays show the direct arrivals from the surface interferers. The bright lines with similar shape above and below are arrivals from bottom reflections (lower portion of figure) and multiple surface/bottom reflections. The red overlay shows the trace of the submerged target and it is only partially visible due to the loud surface interferers. The beam output at the angles/times corresponding to the trace overlays in (b) was used to perform the depth-based transform. 
to the problem at hand is discussed.

\subsection{Literature Review of Source Localization Methods}

This section is a brief discussion of three prevalent underwater source localization methods in the literature. The first, multi-path ranging, is a technique that provides a conceptual framework for approaching the problem at hand but is otherwise not directly applicable. The second, the waveguide invariant, primarily provides range information for broadband sources, but range is already had for signals resolved in vertical angle on the deep VLA, and the target signals are often narrowband (Maranda, 2008).

The third technique is matched field processing. It uses an assumed model of the ocean to attempt to match the received signals with a modelled source at a specific location. It relies on an accurate (complex) model of the ocean, which is its primary limitation (Baggeroer et al., 1993). In contrast, depth estimation using the depth based signal separation method utilizes a simple model of a point source in a uniform sound speed half space. This is possible due to the primary use of the minimally refracted direct and surface-reflected arrivals at the VLA.

\section{Multipath Ranging}

Localization using multi-path ranging is based on using simple geometric relationships to determine the range and depth of targets (Bangs and Schultheiss, 1973). At a sonar receiver (in the active case), multiple backscattered sonar pings are recorded 
that travel distinct paths from the target to the receiver. The relative delays between pings provides information as to the relative path lengths of each arrival and the ensemble delay gives the mean range to the target. From the relative delays the depth may be determined. In the case of a longer, known signal such as pseudorandom noise, pulse-compression processing may be used to determine the arrival directions and delays. Passive ranging on an array is possible (Hassab and Boucher, 1979), though the range uncertainty in the passive and active case is comparable to depths of submerged sources (Carter, 1979). One additional, related technique is to measure wavefront curvature. This provides an estimate of range, but not depth.

\section{Passive Ranging Using the Waveguide Invariant}

The waveguide invariant describes the complex interactions between the multi-path arrivals of continuous signals (Chuprov, 1982). If viewed on a spectrogram, the signals from a moving source will exhibit an interference pattern that has the appearance of sloped lines across the plot (at least in shallow water). The slope of these patterns is related to the relative motion and range of the source. This was demonstrated by (D'Spain and Kuperman, 1999). As range is already given by beamforming on a deep VLA, it could be considered that the interference pattern seen on narrowband signals from moving, submerged sources is a part of the overall pattern described by the waveguide invariant, though only if the target changes depth. However, the general technique is not readily applicable to depth estimation of quiet sources as the received signals are not broadband and the waveguide invariant cannot be readily determined due to environmental uncertainty. 


\section{Matched Field Processing}

Matched field processing (MFP) is the modelling and prediction of sound sources at many depths and ranges in a search to match up the predicted signals for a given location to the real received signals on the array. A comprehensive review paper (Baggeroer et al., 1993) discusses the technique and its limitations, especially that it is sensitive to an incorrect model of the ocean environment.

For the simulations presented in this work, MFP would provide an accurate depth estimate. However, as previously stated and as will be reviewed in the next chapter, a full model of the ocean is not required to predict the interference structure of received signals from a submerged target in RAP geometries (McCargar and Zurk, 2012, 2013). As a full model is not required and that MFP is sensitive to model errors in the real ocean, matched field processing is not used in the analysis presented in this thesis.

\subsection{Conclusion}

The fundamentals of plane-wave beamforming on an uniform line array have been reviewed as well as how its time-varying output is used to perform detection and tracking of submerged sources. Then, the basics of acoustic propagation in the deep ocean was examined. Simulation results showing the signals, of targets, interferers and noise, received on a deeply deployed vertical line array were presented and discussed. Finally, several other underwater source localization techniques were discussed and it was presented that those techniques are either not directly applicable or not suited to the problem at hand, and in some cases, may be far too complicated and involved 
when compared with the simplicity of the propagation physics of the topic of this thesis, as previous authors have shown (McCargar and Zurk, 2013). 


\section{Chapter 3}

\section{Depth-Harmonic Structure Theory}

Figure 3.1 illustrates the problem geometry, wherein a VLA near the ocean bottom, centered at depth $\bar{z}$, is insonified by a submerged source at constant depth $z_{s}$ at a surface range $r$ emitting a tone at frequency $\omega=2 \pi f$ with complex spectral amplitude $S(\omega)$. The slant ranges from the direct and surface-reflected paths of the submerged source and its image are given by $R^{+}$and $R^{-}$, respectively. From image theory, the pressure on the VLA is given by

$$
p(z, \omega)=S(\omega)\left[\frac{e^{i k R^{+}}}{R^{+}}-\frac{e^{i k R^{-}}}{R^{-}}\right]
$$

(Jensen et al., 1997) where time dependence $e^{-i \omega t}$ has been suppressed for simplicity, the wavenumber in the environment with sound speed $c$ is given by $k=\omega / c$. While an actual deep ocean environment will likely have a variable sound speed profile and possible bottom reflections, past work (McCargar and Zurk, 2012, Shibley, 2013, McCargar and Zurk, 2013, Zurk et al., 2013) has shown that image theory, which assumes a half space environment with constant sound speed, accurately describes the depth-dependent interference structure from the direct and surface paths - even in more complicated ocean environments. In the deep ocean, the source depth and 


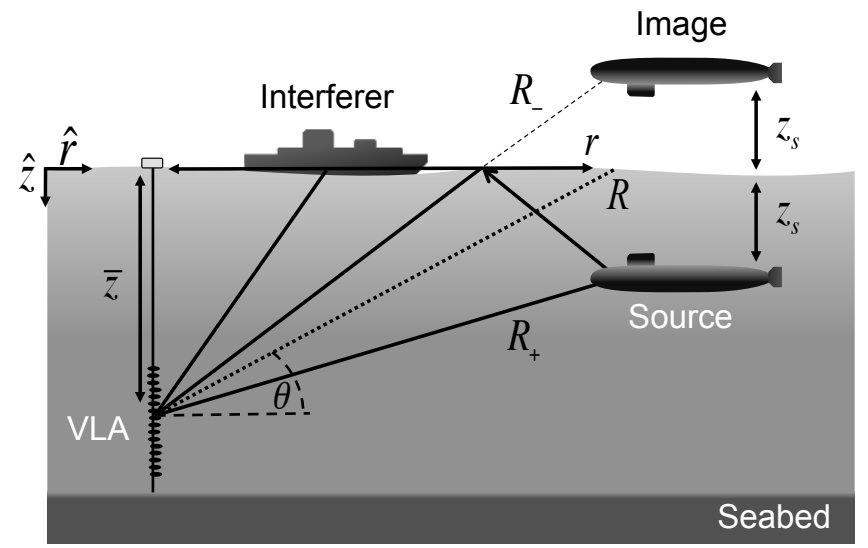

Figure 3.1: The general geometry for received passive signals on a deep vertical line array (not shown to scale). Sound from a submerged source at surface range $r$ and depth $z_{s}$ arrives at an angle of $\theta$ relative to broadside of the VLA whose center lies at depth $\bar{z}$. From image theory, the direct and surface-reflected arrivals correspond to slant ranges $R_{+}$and $R_{-}$, respectively, with slant range $R$ corresponding to the midpoint between the source and its image. Schematic based on Fig. 1 of (McCargar and Zurk, 2013).

the depth of the array center satisfy $\bar{z}>>z_{s}$ and the expression for the complex pressure can be approximated (Jensen et al., 1997) as

$$
p(z, \omega) \approx-2 i S(\omega) \frac{e^{i k R}}{R} \sin \left(k z_{s} \sin \theta\right)
$$

where $R=\sqrt{\bar{z}^{2}+r^{2}}$ is the slant range to the vertical midpoint between the source and its image and $\theta$ is the vertical angle to the target as shown in Figure 3.1.

For a target traveling on a straight line course past the array at constant velocity $v$, the vertical arrival angle of its acoustic signal is related to its position by

$$
\sin \theta(t)=\frac{\bar{z}}{\sqrt{\bar{z}^{2}+r_{C P A}^{2}+v^{2}\left(t-t_{C P A}\right)^{2}}},
$$

where $r_{C P A}$ and $t_{C P A}$ are the surface range and time at the target's closest point of 
approach (CPA). For a bottom-mounted VLA with sufficient vertical aperture, the arrival angle $\theta$ can be determined using a plane-wave beamformer. The time-varying output of the beamformer is referred to as a vertical time record (VTR), analogous to a bearing time record (BTR) - the time-varying output of horizontal line array beamformer.

The received time-domain signal on the array is windowed into snapshots of length $\Delta t$, to which a FFT is applied. Then, conventional beamforming is performed in vertical angle. Each time step in the VTR image is the output of the conventional beamformer for each data snapshot, which shows $\max$ output at $\sin \theta$ corresponding approximately to (3.3). In a real application, such traces along a target track may be selected manually by a sonar operator or automatically by a detection/tracking algorithm.

Once a target trace has been determined, the beamformer output along the trace yields a trace signal, which from (3.2) can be expressed as (McCargar and Zurk, 2012, Shibley, 2013, McCargar and Zurk, 2013, Zurk et al., 2013)

$$
B(\omega, \sin \theta)=2 \frac{|S(\omega)|^{2}}{R^{2}}\left[1-\cos \left(2 k z_{s} \sin \theta\right)\right] .
$$

The cosine term in (3.4) encapsulates the depth-dependent modulation, which is periodic in the sine of vertical arrival angle $(\sin \theta)$ as shown in Figure 3.2(b). However, the spatial distribution of this interference pattern places constraints on target trajectories that allow it to be observed sufficiently for depth estimation over the exert of a target's track. These constraints are analyzed in greater detail in the following section. 

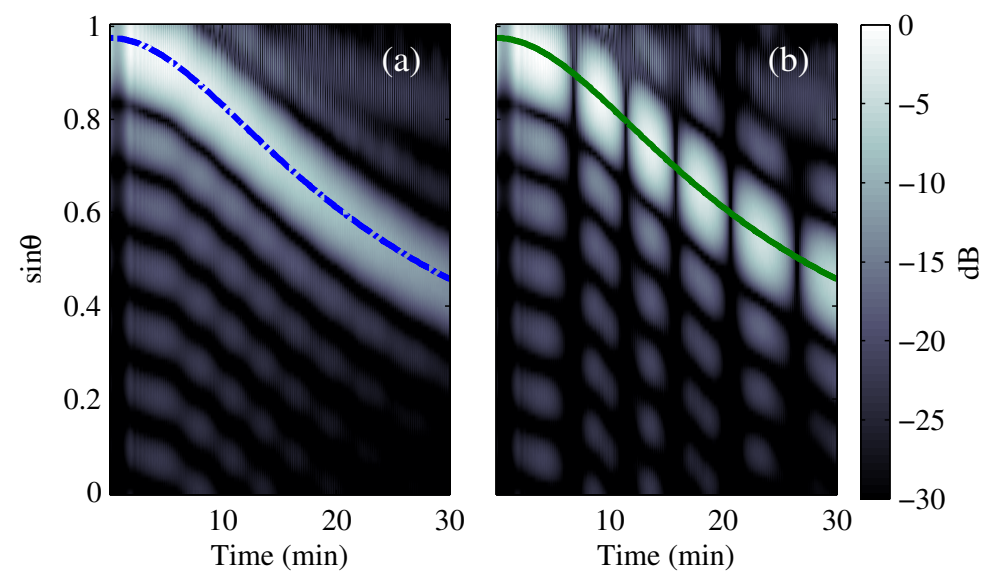

Figure 3.2: Example of depth-dependent modulation in a VTR at $150 \mathrm{~Hz}$ for source depth of $1 \mathrm{~m}$ (a) and $50 \mathrm{~m}$ (b). The null spacing is proportional to frequency and depth (Eq. 3.2).

Figure 3.2 shows two example VTRs generated using data from normal-mode (KRAKEN (Jensen et al., 1997)) simulations for two source depths: $z_{s}=1 \mathrm{~m}$ in Figure 3.2(a) and $z_{s}=50 \mathrm{~m}$ in Figure 3.2(b). Both cases assume a noiseless environment with a refracting Munk sound speed profile (Munk, 1974) and a single target traveling on a straight line course past the array at constant velocity $v$ with range and time at the closest point of approach (CPA) given by $r_{C P A}$ and $t_{C P A}$, respectively. These and all other parameters used in generating the simulation data shown in Figure 3.2 are listed in Table 3.1.

The target tracks previously mentioned are illustrated by the traces plotted as dashed and solid lines in Figure 3.2(a) and (b), respectively, which were generated using (3.3) with a small downward shift in $\sin \theta$ to correct for the small amount of refraction introduced by the Munk profile.

The beamformer output taken along the traces in Figure 3.2 is shown in Figure 3.3. The presence of the interference structure in the trace signal automatically indi- 


\begin{tabular}{|l|c|l|}
\hline Description & Symbol & Value \\
\hline \hline Source frequency & $f$ & $150 \mathrm{~Hz}$ \\
\hline Source level & SL & $110 \mathrm{~dB}$ re $1 \mu \mathrm{Pa}$ \\
\hline Source depth & $z_{s}$ & $\begin{array}{l}\text { (a) } 1 \mathrm{~m} \\
\text { (b) } 50 \mathrm{~m}\end{array}$ \\
\hline Surface range at source CPA & $r_{C P A}$ & $20 \mathrm{~m}$ \\
\hline Time at source CPA & $t_{C P A}$ & $0 \mathrm{~s}$ \\
\hline Source velocity & $v$ & $5 \mathrm{~m} / \mathrm{s}$ \\
\hline Array center depth & $\bar{z}$ & $4972.5 \mathrm{~m}$ \\
\hline Time snapshot length & $\Delta t$ & $3.6 \mathrm{sec}$ \\
\hline Array elements & $N$ & 10 \\
\hline Array spacing & $d$ & $5 \mathrm{~m}$ \\
\hline Water column depth & - & $5000 \mathrm{~m}$ \\
\hline
\end{tabular}

Table 3.1: Normal mode (KRAKEN) simulation parameters used in generating VTR data shown in Figure 3.2.

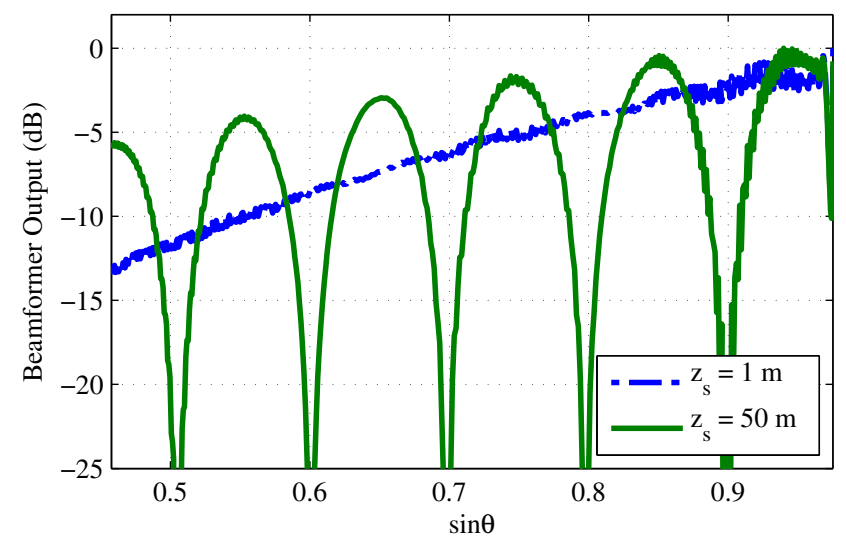

Figure 3.3: Example of depth-dependent modulation in trace signals from the $150 \mathrm{~Hz}$ source at 50 and $1 \mathrm{~m}$ depth shown in the VTRs in Figure 3.2. 
cates a target at depth. This depth may be estimated by measuring the interference null spacing in $\sin \theta$, as will be described later, or by using the recently-developed transform-based method (McCargar and Zurk, 2012, 2013, Zurk et al., 2013), which involves more complicated processing but is likely to be more useful for automated discrimination between surface and submerged contacts.

This transform of the trace signal (McCargar and Zurk, 2012, 2013, Zurk et al., 2013) can be expressed in a closed form by expressing $\sin \theta$ in terms of the vertical wavenumber (Gabriel Kniffin, personal communication),

$$
k_{z}=k \sin \theta,
$$

resulting in

$$
M(z, \omega)=\int_{\text {trace }} B\left(k_{z}\right) e^{i k_{z} z} d k_{z},
$$

where $B\left(k_{z}\right)=B(\omega, \sin \theta)$ in (3.4) for $\omega=k c$ and $\sin \theta=k_{z} / k$. Applying this transform to the trace signals in Figure 3.3 yields the outputs shown in Figure 3.4. The peaks in the transform output clearly indicate the depth of the target. The large peak at $z=0$ is caused by the slow variation in the trace signal due to geometrical spreading. This can be treated as very low frequency noise and may be removed from the signal by subtracting a line fit to the data, giving the trace signal a mean value of nearly zero. The result of this operation is seen in the lower plot of Figure 3.4. Other Fourier signal analysis techniques such as filters could surely be applied to further improve the observation of peaks in the depth transform outputs.

In the depth transform output, factors such as resolution (the finite width of the depth peaks) and ambiguity (aliasing in the depth estimate) are determined by the 

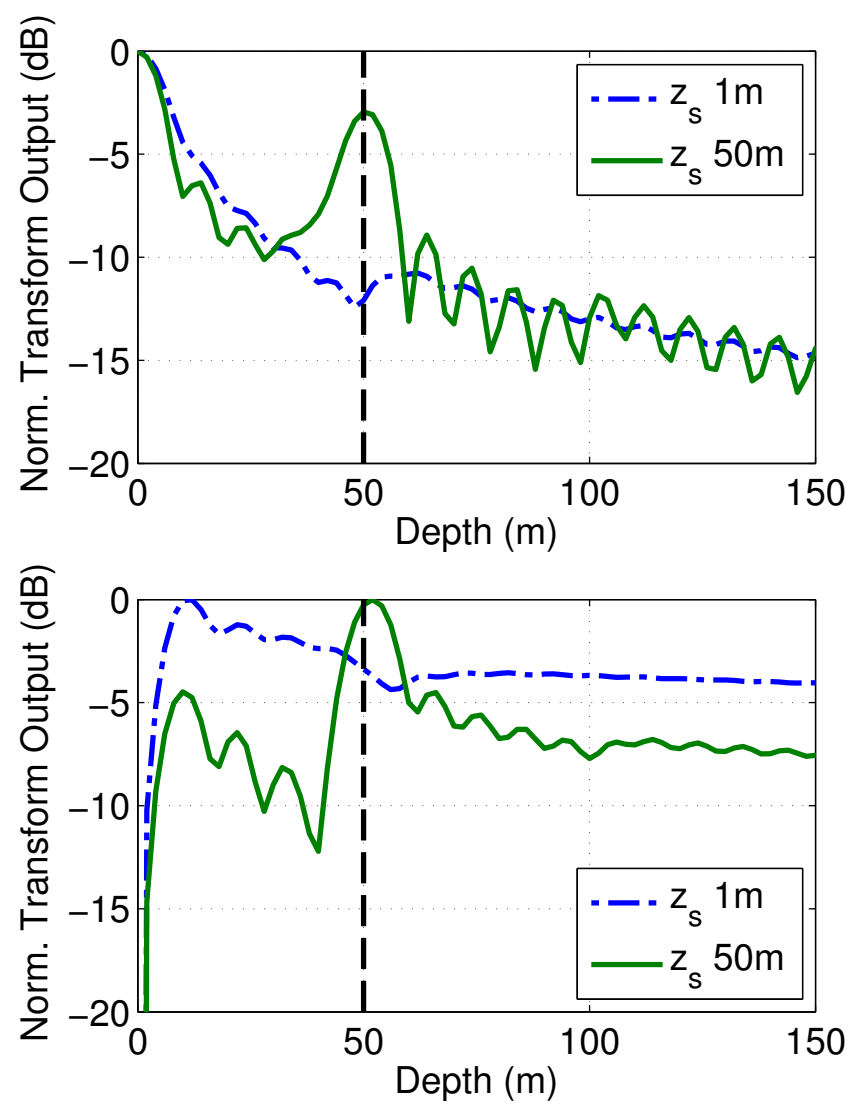

Figure 3.4: Example of tranform-based depth estimation from trace signals shown in Figure 3.3. The upper figure is the transform output of the raw trace signals, the lower is output from the detrended (zero-mean) trace signals. The large peak near $z=0$ is no longer present for neither 1 nor 50 meter depth source signals. The zero-mean signals have slightly different trends in spectra roll-off with frequency than in the original case. However, the peak for the 50 meter source remains clear in the lower plot.

extent and sampling of the trace signal in $k_{z}$, respectively. These factors and their effects on the transform output are characterized in the following sections.

It's important to note that the target trajectory described in (3.3) is a multivalued function - that is, the $\left(t-t_{C P A}\right)^{2}$ term in the denominator results in a symmetry of the target's track in $\sin \theta$ (or equivalently, $k_{z}$ ) about its CPA. In other words, a target's track reaches the same vertical angle twice as it passes through its CPA, 
resulting in two values of $B\left(k_{z}\right)$ for a single $k_{z}$. While the transform may still be applied to the entire signal from a target passing through CPA (McCargar and Zurk, 2012, 2013, Zurk et al., 2013), only one side of the signal (i.e. as the target either approaches or recedes from CPA, but not both) will be considered in the following analysis for simplicity. 


\section{Chapter 4}

\section{Transform-Based Depth Estimation Performance}

A trace signal taken along a target track defined by (3.3), but with a finite angular extent will cause artifacts in the transform output (3.6) that impact resolution and ambiguity in the depth estimate. Their analysis in this chapter is greatly simplified by a formulation developed by Kniffin (personal communication) that describes them with additional factors in Eq. (3.4) prior to application of the modified Fourier transform in Eq. (3.6). The result is

$$
B^{\prime}\left(k_{z}\right)=B\left(k_{z}\right) \Pi\left(k_{z}\right) \amalg\left(k_{z}\right),
$$

where the rectangle function,

$$
\Pi\left(k_{z}\right)= \begin{cases}1 & \text { for } \min \left(k_{z}\right) \leqslant \sin \theta \leqslant \max \left(k_{z}\right) \\ 0 \quad & \text { otherwise }\end{cases}
$$

accounts for the signal's finite extent in $k_{z}$ and $\amalg\left(k_{z}\right)$ is the sampling function (Dirac comb) given by

$$
\amalg\left(k_{z}\right)=\sum_{l} \delta\left(k_{z}-k_{z, l}\right)
$$


where $l$ is the sample time index. Note that the sampling function is a train of Dirac delta functions that is unevenly spaced in $k_{z}$ due to the nonlinear relationship between time and $\sin \theta$ in (3.3).

This nonlinear relationship can be shown explicitly by substituting (3.3) into (3.5) and taking the time derivative, resulting in an approximate relationship between the sampling interval $\Delta t$ in time and in vertical wavenumber $\Delta k_{z}$ along the target trace,

$$
\left.\left.\frac{\Delta k_{z}}{\Delta t}\right|_{\text {trace }} \approx \frac{\partial k_{z}}{\partial t}\right|_{\text {trace }}=\frac{v^{2}\left(t_{C P A}-t\right)}{k^{2} \bar{z}^{2}} k_{z}^{3} .
$$

The result of these two additional factors applied implicitly to the trace signal can be determined by substituting (4.1) into (3.6), resulting in

$$
\begin{aligned}
M(z, \omega)= & \int_{\text {trace }} B^{\prime}\left(k_{z}\right) e^{i k_{z} z} d k_{z} \\
= & A_{M} \int_{\text {trace }}\left[k_{z}^{2} \Pi\left(\frac{k_{z}-\bar{k}_{z}}{K_{z}}\right)\right] \amalg\left(k_{z}\right) \\
& \times\left[1-\cos \left(2 k_{z} z_{s}\right)\right] e^{i k_{z} z} d k_{z} .
\end{aligned}
$$

where the factor $A_{M}=2|S(\omega)|^{2} / k^{2} \bar{z}^{2}$ and $\Pi\left(\frac{k_{z}-\bar{k}_{z}}{K_{z}}\right)$ is (4.2) parameterized in terms of the full trace width

$$
K_{z}=\max \left(k_{z}\right)-\min \left(k_{z}\right),
$$

and center

$$
\bar{k}_{z}=\frac{\max \left(k_{z}\right)+\min \left(k_{z}\right)}{2} .
$$


The net effect of the limited track extent and uneven sampling on the transform output can be most easily interpreted by expressing (4.5) in terms of the threefold convolution,

$$
\begin{aligned}
M(z, \omega)=A_{M} & {\left[\int_{-\infty}^{\infty} k_{z}^{2} \Pi\left(\frac{k_{z}-\bar{k}_{z}}{K_{z}}\right) e^{i k_{z} z} d k_{z}\right] } \\
& *\left[\int_{-\infty}^{\infty} \amalg\left(k_{z}\right) e^{i k_{z} z} d k_{z}\right] \\
& *\left[\delta(z)-\frac{1}{2} \delta\left(z \pm 2 z_{s}\right)\right],
\end{aligned}
$$

wherein the first term represents the effect of the limited track extent (as well as spherical spreading loss), the second term represents the effect of uneven sampling, and the third term is the sum of three delta functions corresponding to depths $z=0$ and $\pm 2 z_{s}$, which is utilized for depth estimation (McCargar and Zurk, 2012, 2013, Zurk et al., 2013). The artifacts described by the first two convolution terms are explored in greater detail in the following sections.

\subsection{Track Extent and Depth Resolution}

The finite, sampled trace signal extent gives rise to the first term in (4.8), which along with the factor of $k_{z}^{2}$ due to spherical spreading loss acts to broaden the delta functions in the transform output. This blurring function can be expressed (McCargar, Kniffin, personal communication) as 


$$
\begin{aligned}
\int_{-\infty}^{\infty} k_{z}^{2} \Pi\left(\frac{k_{z}-\bar{k}_{z}}{K_{z}}\right) & e^{i k_{z} z} d k_{z} \\
& =-K_{z} \frac{\partial^{2}}{\partial z^{2}} e^{i \bar{k}_{z} z} \operatorname{sinc}\left(\frac{K_{z}}{2} z\right),
\end{aligned}
$$

where $\operatorname{sinc}(x)=\sin (x) / x$. The second derivative in the is introduced by the factor of $k_{z}^{2}$ in the integrand, however its full width at half maximum (FWHM) in the $z$ domain is close to that of the sinc function,

$$
z_{F W H M} \approx \frac{1.2 c}{f L_{\sin \theta}}
$$

where $L_{\sin \theta}=K_{z} / k$ is the trace signal extent in $\sin \theta$. This is illustrated in Figures 4.1 and 4.2; reducing the angular extent supplied to the depth transform has the effect of widening the depth peaks occurring at $z=50 \mathrm{~m}$. Figure 4.1 also demonstrates the nonlinear relationship between trace signal extent in time and vertical angle; halving the extent in vertical angle corresponds to a much greater reduction in time.

The expression (4.10) allows setting geometrical constraints that affect the depth resolution in the transform output. For example, the constraint $z_{F W H M}<30 \mathrm{~m}$ would require a $150 \mathrm{~Hz}$ signal from a target at $50 \mathrm{~m}$ depth would need to be received over a portion of its track corresponding to an angular extent of no less than $L_{\sin \theta}=0.2$, or $\theta=11.5^{\circ}$. 


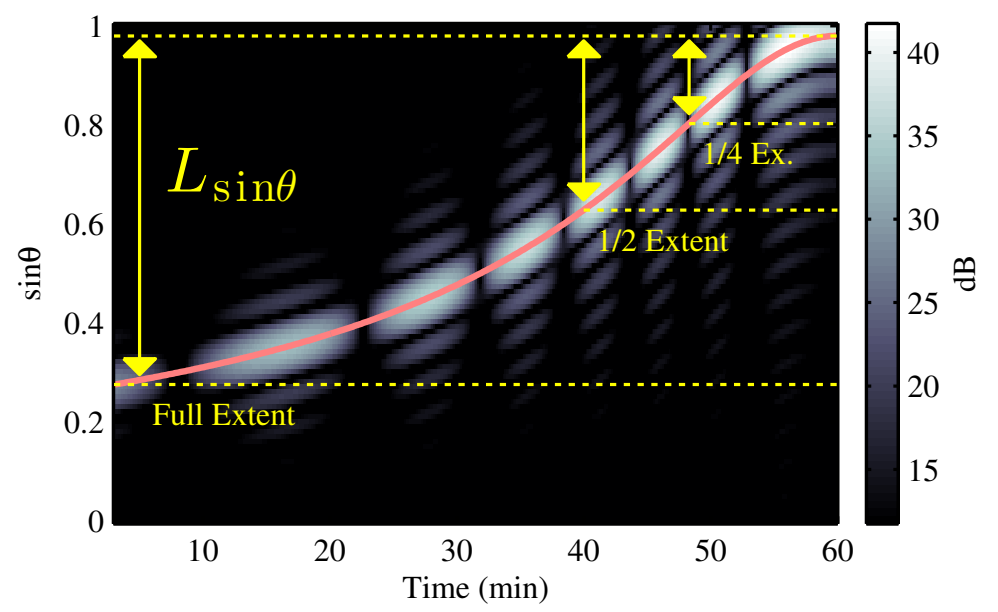

Figure 4.1: Simulated VTR with overlays showing full, half, and quarter extents of the trace signal (in vertical angle), where $L_{\sin \theta}$ is the trace signal extent in $\sin \theta$. Due to the non-linear target track evolution versus time shown in (4.4), the half and quarter length signals (in vertical angle) are much shorter in time than half and quarter of the temporal extent of the VTR. The trace signals corresponding to this VTR are used in the depth transform outputs in Figure 4.2.

\subsection{Trace Signal Sampling and Depth Ambiguity}

The sampling of the trace signal in $k_{z}$ gives rise to the second term in (4.8), which introduces ambiguity in the depth estimate, much like uniform sampling introduces aliasing in a time-harmonic signal (Oppenheim et al., 1999). While this nonuniform "sampling spectrum" is more complicated to express analytically than in the case of uniform sampling, it can be beneficial in that it disrupts the ambiguity that arises from a uniform sampling grid (Marvasti, 1993, Eyer and Bartholdi, 1999).

This is described qualitatively in Figure 4.3. The Fourier transform of the sampled trace signal shown exhibits noisy aliasing due to the uneven sampling. If the sampling were uniform, the three peaks in the center of the lower plot would be repeated at the harmonics of the sampling frequency (aliasing). Instead, the nonuniform spatial 


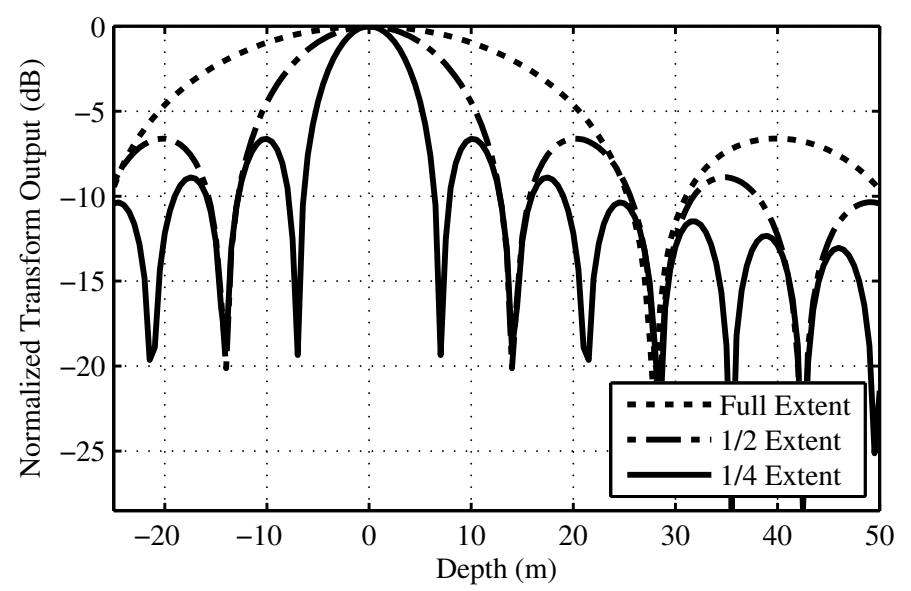

Figure 4.2: Depth transform outputs for the three trace signal extents shown in Figure 4.1, where full, half, and quarter refer to the $\sin \theta$ trace signal extent relative to the full signal in the VTR. As expected, the FWHM is inversely proportional to the trace signal extent.

sampling causes a reduction and smearing of the alias peaks. The depth at which this occurs is given by

$$
z_{\text {alias }}=\frac{c}{4 f \max [\Delta \sin \theta]},
$$

where $\max [\Delta \sin \theta]$ is the maximum sampling interval in $\sin \theta$ for a given trace, corresponding to the greatest angular movement between successive time snapshots along the trace. In the case of uniform sampling in $\sin \theta$, this expression reduces to the traditional expression for the Nyquist depth. The maximum sampling interval is dependent on the target track evolution in $\sin \theta$, the temporal sampling, and other additional processing such as overlapping snapshots to increase SNR.

The effective alias (Nyquist) depths for two frequencies are shown in Table 4.1 for the target traces corresponding to the trace signals in Figure 3.3. Once a target trace is selected in the VTR (as in scissorgram processing), the alias depth can be determined using (4.11). 


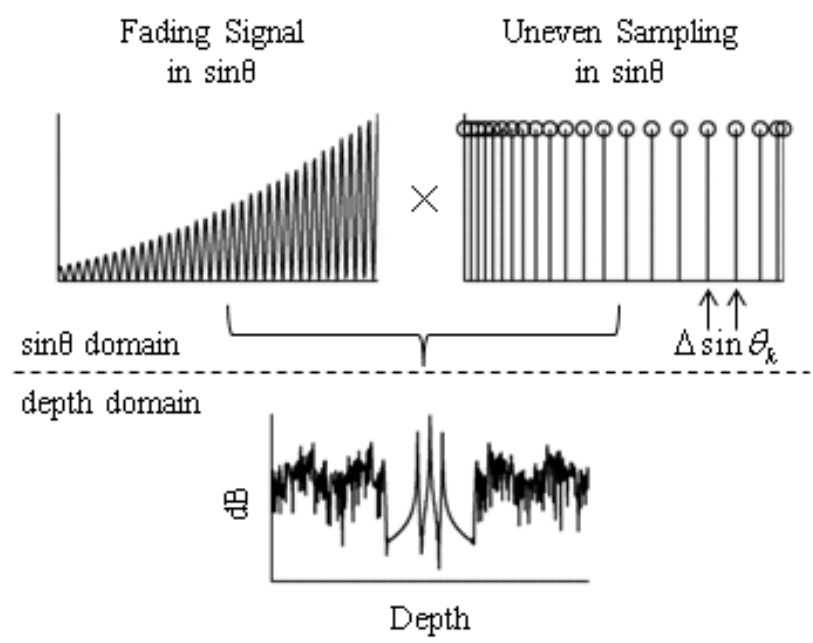

Figure 4.3: Diagram illustrating the effect of irregular sampling in $\sin \theta$ on depth transform output. The signal in $\sin \theta$ and the sampling function are shown (upper left and right). The product of the two may represented in the depth domain by the lower plot. The three peaks near the center of the lower plot represent the three delta functions from the third term in (4.8). The irregular sampling causes noisy aliasing with reduced level relative to the true peaks. This may permit resolving peaks at depths beyond the noisy alias point.

Given the alias depth (4.11), the upper limit on the snapshot length for a given target track, depth, and frequency can be expressed as

$$
\frac{z_{\text {alias }}}{z_{s}}=\frac{\Delta t_{\max }}{\Delta t},
$$

where $\Delta t$ is the slow time snapshot length used in the short-time Fourier transform (not the time signal sample period) and $\Delta t_{\max }$ is the longest possible snapshot length that will not lead to aliasing of the target peak in the depth transform output. It is the ratio of the snapshot length related to the effective alias depth and the current data snapshot length. Common snapshot lengths of several seconds are sufficient for depth separation of low frequency, slow moving targets. 


\begin{tabular}{|c|c|}
\hline \multicolumn{2}{|c|}{ Nyquist Depths for Submerged Target at 50m } \\
\hline Frequency $\mathbf{( H z )}$ & Nyquist Depth \\
\hline 100 & $1.4 \mathrm{~km}$ \\
\hline 200 & $700 \mathrm{~m}$ \\
\hline
\end{tabular}

Table 4.1: Alias (effective Nyquist) depths for the target traces corresponding to the trace signals in Figure 3.3 calculated using (4.11) at two frequencies (the simulation parameters are described in the description of Figure 3.2). Depth ambiguity points are inversely proportional to the frequency at which the trace signal is taken. The Nyquist depth of the higher frequency trace signal is therefore lower. 


\section{Chapter 5}

\section{Estimating Depth by Measuring Null Spacing}

As previously mentioned, the presence of an interference structure in a trace signal indicates a target at depth, and the nulls in the trace signal (3.4) occur when the target is at vertical angles that satisfy

$$
\sin \theta_{m}=\frac{m c}{2 f z_{s}}, \quad m=1,2,3, \ldots
$$

(Jensen et al., 1997) where $\theta_{m}$ is the location of the m-th null. By taking the difference of the expressions for two adjacent null, the corresponding source depth is found by

$$
z_{s}=\frac{c}{2 f \sin \Delta \theta}
$$

where $\Delta \theta$ is the interval between two adjacent nulls. Thus, given nulls visible in a VTR along a track, depth may be estimated without using the full depth transform, nor is a long, uncluttered trace signal required.

This simple method of measuring null spacing does not require the application and interpretation of the depth transform and its output. Its simplicity may be of advan- 
tage in a VTR cluttered by many surface interferers; only a small number of nulls might be visible between the overlapping tracks.

In the next section, the ability to accurately measure the null period will be discussed as it relates to array aperture, and a worst-case depth-estimate error analysis will be performed.

\subsection{Aperture Requirements}

The depth-harmonic interference pattern of a submerged source will be observed on any aperture, including a single hydrophone, provided the signal is received with sufficient signal-to-noise ratio (SNR) for detection. However, as indicated by (3.4), the interference pattern is periodic in the sine of its vertical arrival angle, which a single hydrophone cannot determine due to its lack of vertical aperture. Furthermore, an increase in vertical aperture (number of elements in the VLA) increases the SNR of the received depth-harmonic signal as well as the vertical angular resolution, both of which improve the accuracy of the resulting depth estimate. Conversely, shorter apertures reduce the SNR and degrade the resolution in the vertical arrival angle estimate, which in turn reduces the accuracy of the depth estimate. In the following analysis, the angular resolution is assumed to be the limiting factor that imposes a minimum number of hydrophones in the array. The accuracy in vertical angle estimation is therefore quantified using the $3 \mathrm{~dB}$ beam width in this analysis.

Figure 5.1 shows the VTR of a $150 \mathrm{~Hz}$ source at $z_{s}=50 \mathrm{~m}$ depth from a 10-element VLA. A clear null at $\sin \theta=0.9$ is observed, and in this analysis, the location of the null at $\sin \theta=0.8\left(\approx 53^{\circ}\right)$ is by an amount equal to half the array's $3 \mathrm{~dB}$ beam 


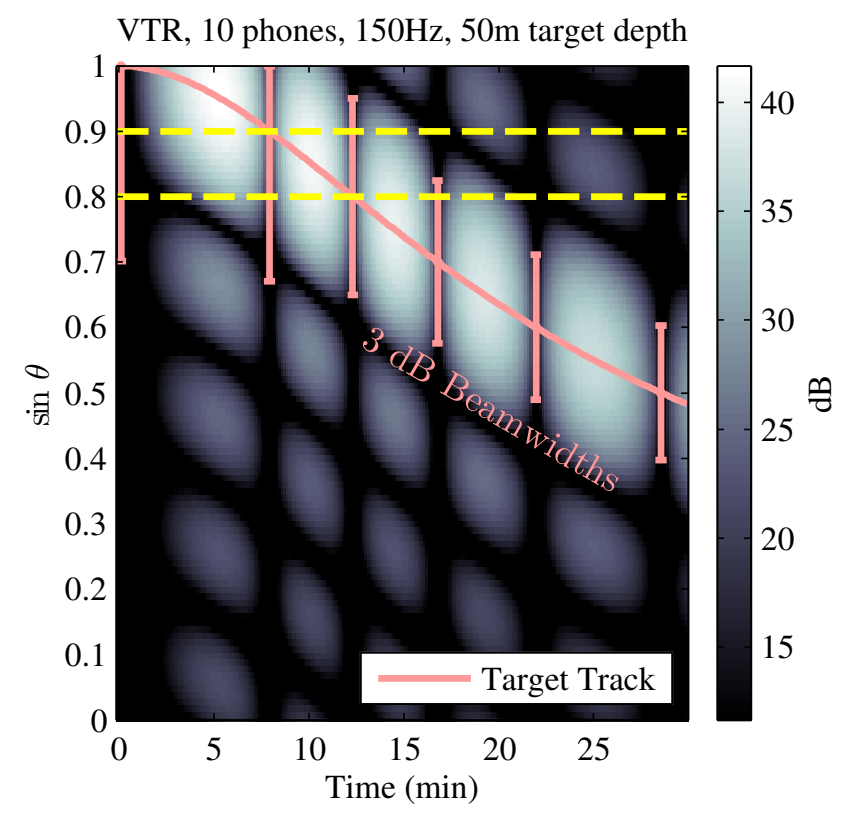

Figure 5.1: Illustration of using the $3 \mathrm{~dB}$ beamwidth to estimate the worst-case depth estimate. The simulated VTR was obtained an image theory simulation for a $150 \mathrm{~Hz}$ source at $50 \mathrm{~m}$ depth received on a 10-element VLA. The dashed horizontal lines indicate the positions in $\sin \theta$ of two nulls, while the the true target track is shown as a solid line with error bars corresponding to the $3 \mathrm{~dB}$ beamwidth.

width (indicated by the vertical error bars) in the corresponding look direction for the array. The error in the depth estimate is calculated by introducing \pm half of the $3 \mathrm{~dB}$ beamwidth $\theta_{3 d B}$ into (5.2), resulting in

$$
z_{s}=\frac{c}{2 f\left[0.9-\sin \left(53^{\circ} \pm \frac{\theta_{3 d B}}{2}\right)\right]}
$$

the bounds of which are plotted in Figure 5.2 along with the ground truth.

From Figure 5.1, the target track appears to be easily identifiable visually and may accurately be estimated manually or using a Bayesian tracking algorithm or other method. Thus, this tracking error based only on the array's $3 \mathrm{~dB}$ beam width is likely to be a pessimistic worst-case scenario. Even so, while depth estimates with 


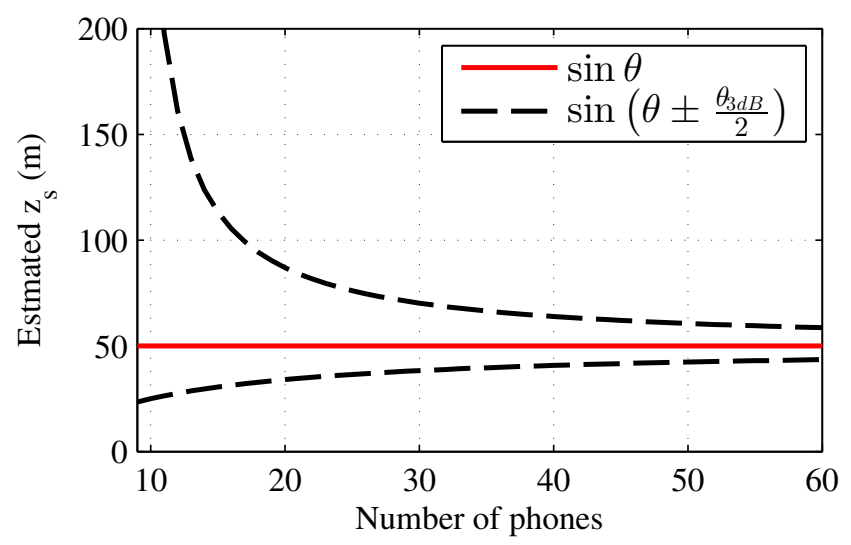

Figure 5.2: Error in depth estimate using (5.3), corresponding to the null at $0.9 \sin \theta$ in Figure 5.1 and an erroneous value for the null at $0.8 \sin \theta$ as given by the $3 \mathrm{~dB}$ beamwidth, plotted as a function of number of phones. Ground truth $\left(z_{s}=50 \mathrm{~m}\right)$ is given by the solid line, while error bounds corresponding to an error of $\pm \theta_{3 d B} / 2$ in null location are given by the dashed lines. The lower error bound shows that even in error, the estimated depth is at worst $25 \mathrm{~m}$ for this situation. The errors given by the upper bound line are not critical as the key concern is if a observed target is submerged or at the surface. Thus, errors toward 0 meters depth are more complicating.

small arrays may lose accuracy, the presence of the depth-harmonic interference is still likely to be observable and indicate a submerged source without the need for a great degree of accuracy in estimated depth. 


\section{Chapter 6}

\section{Summary}

This thesis provides a detailed analysis of the factors impacting the performance of a recently introduced transform-based method for estimating the depth of submerged targets by VLAs positioned below the critical depth in the deep ocean. Such deep VLAs have been recently shown to utilize favorable propagation conditions that suppress noise from distant interferers and reduce transmission loss from sources at moderate ranges. This transform-based method exploits the nearly ubiquitous, depthdependent Lloyd's mirror interference pattern, which arises from the interaction of direct and surface-reflected acoustic arrivals. The observation of this depth-harmonic modulation is dependent on the VLA beamformer's resolution. VLAs with as few as 10 elements (assuming sufficient SNR) are likely to be able to resolve the interference structure sufficiently to accurately distinguish surface interferers from submerged targets even if an accurate depth estimate cannot be obtained. The performance characterization of the transform-based method included the derivation of a simple expression for the depth resolution in the transform output in terms of the extent of the target's track in vertical angle. The resolution in depth shows that a $30 \mathrm{~m}$ resolution (FWHM) in the transform output for a target at $50 \mathrm{~m}$ emitting a $150 \mathrm{~Hz}$ tone can be achieved with a target track encompassing as little as $11.5^{\circ}$ of angular extent. 
In addition, the nonuniform sampling imposed by the collection geometry limits the ambiguity (aliasing) in the depth estimate to depths on the order of thousands of meters, making the ambiguity negligible for slow, low-frequency targets. 


\section{Bibliography}

Arthur B Baggeroer, William A Kuperman, and Peter N Mikhalevsky. An overview of matched field methods in ocean acoustics. Oceanic Engineering, IEEE Journal of, 18(4):401-424, 1993.

William J Bangs and Peter M Schultheiss. Space-time processing for optimal parameter estimation. Signal Processing, pages 577-590, 1973.

AV Belousov and AV Furduev. Estimation of the noise-level in the ocean in the angular interval of the refraction minimum. SOVIET PHYSICS ACOUSTICSUSSR, 34(4):421-421, 1988.

L.M. Brekhovskikh and Yu.P. Lysanov. Fundamentals of Ocean Acoustics, 3rd ed. Springer-Verlag New York, Inc, 2003. ISBN 0387954678.

G Clifford Carter. Passive ranging errors due to receiving hydrophone position uncertainty. The Journal of the Acoustical Society of America, 65(2):528-530, 1979.

SD Chuprov. Interference structure of a sound field in a layered ocean. Ocean Acoustics, Current State, pages 71-91, 1982.

G.L. D'Spain and W.A. Kuperman. Application of waveguide invariants to analysis of spectrograms from shallow water environments that vary in range and azimuth. The Journal of the Acoustical Society of America, 105:2454-2468, 1999.

Rui Duan, Kunde Yang, Yuanliang Ma, Qiulong Yang, and Hui Li. Moving source localization with a single hydrophone using multipath time delays in the deep ocean. The Journal of the Acoustical Society of America, 136(2):EL159-EL165, 2014.

L Eyer and P Bartholdi. Variable stars: Which nyquist frequency? Astron. Astrophys. Suppl. Ser, 135:1-3, 1999.

R.D. Gaul, D.P. Knobles, J.A. Shooter, and A.F. Wittenborn. Ambient noise analysis of deep-ocean measurements in the northeast pacific. IEEE Journal of Oceanic Engineering, 32(2):497 -512, apr. 2007. ISSN 0364-9059. doi: 10.1109/JOE.2007.891885. 
JC Hassab and RE Boucher. Passive ranging estimation from an array of sensors. Journal of Sound and Vibration, 67(2):289-292, 1979.

Finn B. Jensen, William A. Kuperman, Michael B. Porter, and Henrik Schmidt. Computational Ocean Acoustics. American Inst. of Physics, 1997. ISBN 1563962098.

Zizheng Li, L.M. Zurk, and B. Ma. Vertical arrival structure of shipping noise in deep water channels. In OCEANS 2010, pages 1 -8, sept. 2010.

Brian H. Maranda. Passive sonar. In Handbook of Signal Processing in Acoustics, Vol 1, page 1761. Springer Science+Business Media, LLC, 2008.

Farokh Marvasti. Nonuniform sampling. In Advanced topics in Shannon sampling and interpolation theory, pages 121-156. Springer, 1993.

Reid McCargar and Lisa M. Zurk. Depth-based signal separation with vertical line arrays in the deep ocean. The Journal of the Acoustical Society of America, 133(4):EL320-EL325, 2013. doi: http://dx.doi.org/10.1121/1.4795241. URL http://scitation.aip.org/content/asa/journal/jasa/133/4/10.1121/1.4795241.

Reid K McCargar and Lisa M Zurk. Depth-based suppression of moving interference with vertical line arrays in the deep ocean. The Journal of the Acoustical Society of America, 132(3):2081-2081, 2012.

Walter H Munk. Sound channel in an exponentially stratified ocean, with application to sofar. The Journal of the Acoustical Society of America, 55(2):220-226, 1974.

Alan V. Oppenheim, Ronald W. Schafer, and John R. Buck. Discrete-time Signal Processing, 2nd ed. Prentice-Hall, Inc, 1999. ISBN 0137549202.

Henrik Schmidt. OASES Version 3.1, 2011.

Jordan A. Shibley. Enhanced sonar array target localization using time-frequency interference phenomena. Master's thesis, Portland State University, Portland, OR, 2013

Petre Stoica and Randolph L Moses. Spectral analysis of signals. Pearson/Prentice Hall Upper Saddle River, NJ, 2005.

Robert J. Urick. Principles of Underwater Sound 3rd Edition. Peninsula Pub, August 1996. ISBN 0932146627.

Gordon M Wenz. Acoustic ambient noise in the ocean: spectra and sources. The Journal of the Acoustical Society of America, 34(12):1936-1956, 1962.

Lisa M Zurk, Nigel Lee, and James Ward. Source motion mitigation for adaptive matched field processing. The Journal of the Acoustical Society of America, 113 (5):2719-2731, 2003. 
Lisa M Zurk, John K Boyle, and Jordan Shibley. Depth-based passive tracking of submerged sources in the deep ocean using a vertical line array. In Signals, Systems and Computers, 2013 Asilomar Conference on, pages 2130-2132. IEEE, 2013. 